\title{
Principais fatores relacionados ao risco cardiovascular de Populações Indígenas do
}

\section{Brasil}

\author{
Main factors related to cardiovascular risks for Indigenous Populations of Brazil \\ Principales factores relacionados com los riesgos cardiovasculares para las Poblaciones Indígenas \\ de Brasil
}

Recebido: 14/07/2021 | Revisado: 20/07/2021 | Aceito: 21/07/2021 | Publicado: 28/07/2021

\author{
Ilton Palmeira Silva \\ ORCID: https://orcid.org/0000-0003-4135-5767 \\ Centro Universitário do Rio São Francisco, Brasil \\ E-mail: Ilton.p@hotmail.com \\ João Ricardhis Saturnino de Oliveira \\ ORCID: https://orcid.org/0000-0002-5024-8253 \\ Universidade Federal de Pernambuco, Brasil \\ E-mail: ricardhis@gmail.com \\ Bianka Santana dos Santos \\ ORCID: https://orcid.org/0000-0003-3313-039X \\ Universidade Federal de Pernambuco, Brasil \\ E-mail: bianka.santos@ufpe.br \\ Caíque Silveira Martins da Fonseca \\ ORCID: https://orcid.org/0000-0002-6812-3811 \\ Faculdade Tiradentes de Jaboatão dos Guararapes, Brasil \\ E-mail: caiquesmfonseca@gmail.com \\ Vera Lúcia de Menezes Lima \\ ORCID: https://orcid.org/0000-0003-2581-9218 \\ Universidade Federal de Pernambuco, Brasil \\ E-mail: lima.vera.ufpe@gmmail.com
}

\begin{abstract}
Resumo
A população brasileira de indígenas vem passando por um processo de mudança no perfil de suas doenças mais prevalentes. As doenças infecto-parasitárias têm perdido o pódio para doenças crônicas não transmissíveis. Dentre elas, as doenças cardiovasculares são as de maior aumento na prevalência, o que aumenta riscos para eventos que possam comprometer a qualidade de vida e saúde destes grupos étnicos. Pouco se sabe sobre os riscos cardiovasculares que a população indígena brasileira apresenta, pois poucos estudos visam estes grupos. Logo, esta revisão objetivou rastrear os principais fatores reportados. De acordo com a literatura, a urbanização foi o fator desencadeador do aparecimento de doenças cardiovasculares, gerando sedentarismo e mudanças nos hábitos alimentares. Estes foram reportados como principais causas no aumento do número de indivíduos com obesidade, hipertensão e dislipidemias. Diabetes mellitus tipo 2 ainda é pouco frequente em indivíduos sem outras comorbidades, porém a prevalência aumenta em cinco vezes quando há alguma doença associada. Portanto, os grupos étnicos brasileiros necessitam de mais suporte educacional e de saúde para prevenir e tratar doenças crônicas não transmissíveis, e é necessária maior preocupação em acompanhar as incidências destas condições dentro de territórios indígenas.
\end{abstract}

Palavras-chave: Indígenas; Doenças cardiovasculares; Urbanização; Síndrome metabólica.

\begin{abstract}
The Brazilian indigenous population has been going through a process of change in the profile of its most prevalent diseases. Thus, infectious and parasitic diseases have lost the podium for chronic non-communicable diseases. Among them, cardiovascular diseases are the ones with the greatest increase in prevalence, which increases the risk of events that could compromise the quality of life and the health of these ethnic groups. Little is known about the cardiovascular risks that the Brazilian indigenous population presents, as few studies target these groups. Therefore, this review aimed to track the main factors reported in the literature. We have observed the urbanization was the triggering factor for the onset of cardiovascular diseases, generating a sedentary lifestyle and changes in eating habits. These factors have been reported as the main causes of the increase in the number of individuals with obesity, hypertension and dyslipidemia. Type 2 Diabetes mellitus is still uncommon in individuals without other comorbidities, but its prevalence increases fivefold when there is an associated disease. Therefore, Brazilian ethnic groups need more educational and health support to prevent and treat chronic non-communicable diseases, and greater concern is needed to monitor the incidence of these conditions within indigenous territories.
\end{abstract}


Keywords: Indigenous; Cardiovascular diseases; Urbanization; Metabolic syndrome.

\section{Resumen}

La población indígena brasileña ha atravesado un proceso de cambio en el perfil de sus enfermedades más prevalentes. Las enfermedades infecciosas y parasitarias han perdido el podio ante las enfermedades crónicas no transmisibles. Entre ellas, las enfermedades cardiovasculares son las de mayor aumento de prevalencia, lo que aumenta el riesgo de eventos que puedan comprometer la calidad de vida y la salud de estos grupos étnicos. Poco se sabe sobre los riesgos cardiovasculares que presenta la población indígena brasileña, ya que pocos estudios se dirigen a estos grupos. Por lo tanto, esta revisión tuvo como objetivo rastrear los principales factores informados. Según la literatura, la urbanización fue el factor desencadenante de la aparición de enfermedades cardiovasculares, generando sedentarismo y cambios en los hábitos alimentarios. Estos han sido reportados como las principales causas del aumento en el número de individuos con obesidad, hipertensión y dislipidemia. La diabetes mellitus tipo 2 sigue siendo poco común en personas sin otras comorbilidades, pero su prevalencia se quintuplica cuando existe una enfermedad asociada. Por lo tanto, los grupos étnicos brasileños necesitan más apoyo educativo y de salud para prevenir y tratar las enfermedades crónicas no transmisibles, y se necesita una mayor preocupación para monitorear la incidencia de estas condiciones en los territorios indígenas.

Palabras clave: Indígena; Enfermedades cardiovasculares; Urbanización; Síndrome metabólico.

\section{Introdução}

O perfil das doenças que acometem a população indígena brasileira tem se alterado ao longo dos anos, passando a predominar as doenças crônicas não transmissíveis (DCNTs). Destas, destacam-se as doenças cardiovasculares (DCVs), devido ao aumento na prevalência e gastos públicos para prevenção e tratamento (Tavares, Coimbra Jr, Cardoso, 2012; Welch et al., 2009). Embora as doenças infecto-parasitárias ainda possuam altas prevalências em populações indígenas, avanços no saneamento básico têm propiciado diminuição destes números, em um processo denominado de ocidentalização das áreas indígenas. No entanto, a dificuldade de acesso a serviços de saúde, causada por limitações nas políticas públicas e no acesso de agentes de saúde às populações, leva à redução de medidas preventivas contra o aparecimento de distúrbios cardiometabólicos (Sartori, Leivas, 2017; Coimbra Jr, Santos, 2000).

Um dos fatores, entre tantos, que coopera para o processo de ocidentalização e a mudança na epidemiologia de doenças nestes grupos étnicos se explica pelo contato e interações dos indígenas com não indígenas, uma vez que se verifica semelhante tendência de crescimento das referidas morbidades nos vários grupos étnicos distribuídos fora das terras indígenas (Santos, Coimbra Jr, 2003. Leite et al., 2007; Wirsing, 1985). As formas como se deram esses contatos e interações não foram benéficas para as etnias indígenas, visto que o pós-contato provoca mudanças negativas de natureza econômica, social, cultural e ambiental. Notadamente, houve uma drástica diminuição dos territórios tradicionalmente ocupados e explorados pelos indígenas e isto veio a afetar os sistemas de subsistência, ocasionando menor disponibilidade de alimentos e necessidade de busca por outras formas de trabalho (Santos, Coimbra Jr, 2003. Leite et al., 2007).

Devido à dificuldade de realização de estudos com a população moradora de áreas reservadas à população indígena brasileira, é de grande importância o levantamento da bibliografia produzida com informações desta população para um delineamento dos riscos e possibilidades de intervenções para melhoria da saúde destes indivíduos. Apesar de restrita ao Brasil, estas informações também podem despertar preocupação com as populações indígenas de outras áreas do mundo e auxiliar na criação de medidas de preservação à saúde destes grupos étnicos.

\section{Metodologia}

Trata-se de uma revisão integrativa da literatura. Foram utilizadas as bases de dados PubMed, SciELO, Bireme, Web of Science, Cochrane Library e EBSCO; também foi utilizado o Google acadêmico, para busca de artigos nacionais, não indexados, que se enquadrem na temática. Os trabalhos pesquisados foram resultados da seguinte estratégia de busca: junção de dois ou três palavras-chave ou descritores, associados pelo operador booleano AND. Os descritores e palavras-chave 
utilizados em português e inglês foram Indians, Indigenous, Native, Cardiovascular, Cardiovascular risk, Brazil, Brazilian, Prevalence, Incidence, Stroke, Heart attack, Chronic diseases, Cardiometabolic diseases, Dyslipidemia, Obesity, Diet, Nutrition, Hypertension, Arteriosclerosis.

Foram utilizados artigos científicos originais e disponíveis em versão completa para acesso. Apenas os artigos que reportavam características bioquímicas, metabólicas e fisiopatológicas associadas às doenças cardiovasculares de populações indígenas em território brasileiro foram considerados. Não houve exclusão de trabalhos devido ao ano de publicação ou idioma do texto. Os artigos foram encontrados após utilização das palavras-chave. Então, foi realizada triagem por título. Os artigos selecionados tiveram resumo avaliados e checados quanto à disponibilidade do arquivo completo. Após obtenção dos trabalhos completos, os artigos foram lidos na íntegra e apenas os artigos que cumpriam os critérios de inclusão e exclusão foram selecionados para a revisão. Para melhor entendimento, os principais.

\section{Resultados e Discussão}

\section{Achados da Busca}

Ao todo, foram encontrados 926 artigos. Após análise de títulos e resumos, 894 foram excluídos por não estudarem a população indígena brasileira. Dos 32 restantes, que foram avaliados na íntegra, 18 preencheram os critérios de inclusão e compuseram a presente revisão, como mostra a Tabela 1.

Tabela 1 - Estudos realizados com populações indígenas do Brasil sobre fatores preditores de doenças crônicas não transmissíveis e riscos metabólicos.

\begin{tabular}{|c|c|c|c|c|}
\hline Autor & Tipo & População & Principais Achados & Discussão \\
\hline $\begin{array}{l}\text { Armstrong } \\
\text { et al., } 2018\end{array}$ & $\begin{array}{c}\text { Estudo } \\
\text { Transversal }\end{array}$ & $\begin{array}{l}\text { Indígenas das etnias Funi-ô, } \\
\text { Pankararu, Kiriri, Pankararé, } \\
\text { Tuxá, Truká e Tumbalalalá do } \\
\text { Vale do São Francisco, } \\
\text { Pernambuco e Bahia. }\end{array}$ & $\begin{array}{l}\text { Tribos menos urbanizadas } \\
\text { possuem } 15 \% \text { a menos de } \\
\text { risco para óbito por doença } \\
\text { cardiovascular que tribos } \\
\text { urbanizadas. }\end{array}$ & $\begin{array}{l}\text { Mudanças ambientais e no estilo de } \\
\text { vida, além de pouca atenção à saúde } \\
\text { levam a aumento de eventos } \\
\text { cardiovasculares. }\end{array}$ \\
\hline $\begin{array}{l}\text { Fávaro et } \\
\text { al., } 2007\end{array}$ & $\begin{array}{c}\text { Estudo } \\
\text { Transversal }\end{array}$ & $\begin{array}{l}\text { População indígena } \\
\text { aldeias Olho D’Água, Água } \\
\text { Azul e Oliveiras da Ârrea } \\
\text { Indígena do Buriti, Mato } \\
\text { Grosso do Sul. }\end{array}$ & $\begin{array}{l}75,5 \% \text { das } 49 \text { famílias } \\
\text { entrevistadas relataram receio } \\
\text { com a qualidade e quantidade } \\
\text { dos alimentos ingeridos. }\end{array}$ & $\begin{array}{l}\text { A baixa renda per capita gera } \\
\text { insegurança na qualidade dos } \\
\text { alimentos, além de busca por alimentos } \\
\text { pobres em nutrientes por serem mais } \\
\text { baratos. }\end{array}$ \\
\hline $\begin{array}{l}\text { Salvo et al., } \\
2009\end{array}$ & $\begin{array}{c}\text { Estudo } \\
\text { Transversal }\end{array}$ & $\begin{array}{l}\text { Indígenas de etnia Suyá do } \\
\text { Médio Xingu. }\end{array}$ & $\begin{array}{l}\text { Mulheres possuem maior } \\
\text { prevalência de sobrepeso e } \\
\text { obesidade, porém homens } \\
\text { possuem dislipidemia mais } \\
\text { acentuada. }\end{array}$ & $\begin{array}{l}\text { Alterações antropométricas no sexo } \\
\text { feminino se dão pela multiparidade. } \\
\text { Homens tem mais acesso a etilismo e } \\
\text { tabagismo, o que gera alterações } \\
\text { metabólicas. }\end{array}$ \\
\hline $\begin{array}{l}\text { Coimbra Jr } \\
\text { e Santos, } \\
1991\end{array}$ & $\begin{array}{c}\text { Estudo } \\
\text { Transversal }\end{array}$ & $\begin{array}{l}\text { Indígenas de etnia Suruí, do } \\
\text { Parque Indígena Aripuanã, } \\
\text { Rondônia. }\end{array}$ & $\begin{array}{l}\text { Alta prevalência de crianças } \\
\text { com estado nutricional baixo } \\
\text { e sinais de retardo no } \\
\text { desenvolvimento ponderal e } \\
\text { de estatura. }\end{array}$ & $\begin{array}{l}\text { Redução da capacidade de produção de } \\
\text { alimentos e acesso à alimentação } \\
\text { nutritiva alteram o desenvolvimento de } \\
\text { crianças e as deixam com maior } \\
\text { propensão a doenças. }\end{array}$ \\
\hline $\begin{array}{l}\text { Ribas } \\
2001\end{array}$ & $\begin{array}{r}\text { Estl } \\
\text { Trans }\end{array}$ & $\begin{array}{l}\text { População de etnia Terena da } \\
\text { Aldeia Córrego do Meio, } \\
\text { Mato Grosso do Sul. }\end{array}$ & $\begin{array}{l}\text { Mais de } 20 \% \text { das crianças } \\
\text { apresentavam déficit no } \\
\text { desenvolvimento ponderal e } \\
5 \% \text { já apresentavam } \\
\text { obesidade. }\end{array}$ & $\begin{array}{l}\text { Dietas pouco nutritivas e sem controle } \\
\text { de seus componentes gera alterações no } \\
\text { desenvolvimento de crianças e aumenta } \\
\text { o risco para doenças de caráter } \\
\text { metabólico no futuro. }\end{array}$ \\
\hline $\begin{array}{l}\text { Capelli e } \\
\text { Koifman, } \\
2001\end{array}$ & $\begin{array}{r}\text { Estu } \\
\text { Transv }\end{array}$ & $\begin{array}{l}\text { Indígenas de etnia Parkatêjê } \\
\text { do município de Bom Jesus } \\
\text { do Tocantins, Pará. }\end{array}$ & $\begin{array}{l}\text { Mais de } 20 \% \text { dos homens e } \\
\text { mais de } 50 \% \text { das mulheres } \\
\text { possuem sobrepeso. }\end{array}$ & $\begin{array}{l}\text { Apesar de muito relacionado com a } \\
\text { multiparidade, as mulheres também } \\
\text { tiveram maior risco para dislipidemias e } \\
\text { eventos cardiovasculares quanto os } \\
\text { homens, devido a mudanças nos hábitos } \\
\text { de vida, oriundas da urbanização. }\end{array}$ \\
\hline $\begin{array}{l}\text { Gugelmin e } \\
\text { Santos, } \\
2006\end{array}$ & $\begin{array}{c}\text { Estudo } \\
\text { Transversal }\end{array}$ & $\begin{array}{l}\text { População de etnia Xavánte } \\
\text { da Aldeia São José, Mato } \\
\text { Grosso. }\end{array}$ & $\begin{array}{l}\text { Menos de } 25 \% \text { da população } \\
\text { possuíam índice de massa } \\
\text { corpórea adequado. }\end{array}$ & $\begin{array}{l}\text { As altas prevalências de sobrepeso e } \\
\text { obesidade estão relacionadas a aumento } \\
\text { do risco de diabetes, dislipidemias, } \\
\text { hipertensão arterial sistêmica, e eventos }\end{array}$ \\
\hline
\end{tabular}




Mazzucchet
$\begin{aligned} & \text { Coorte } \\ & \text { ti et al., }\end{aligned}$
2014

Indígenas de etnia Suyá do Médio Xingu.

2014

Prospectiva

Gimeno et $\quad$ Estudo

al., $2007 \quad$ Transversal

Indígenas de etnia Mehináku, Waurá, Yawalapití, Kalapálo, Kuikúru, Matipú , Nahukwá, Awetí, Kamayurá, Trumái do Alto Xingu, Mato Grosso.
População de etnia Xavante, moradora das Reservas Indígenas de São Marcos e Sangradouro/Volta Grande, Mato Grosso.

Soares et Estudo

al., 2017 Transversal

localizada entre Planalto e 2011 Transversal Nonoai, Rio Grande do Sul.

Grupo indígena da Aldeia Jaguapiru, Mato Grosso do Sul.

$\begin{array}{ll}\begin{array}{l}\text { Oliveira et } \\ \text { al., 2011 }\end{array} & \begin{array}{c}\text { Estudo } \\ \text { Transversal }\end{array}\end{array}$

\begin{tabular}{lcl} 
& \multicolumn{2}{l}{$\begin{array}{l}\text { Indígenas de etnia Parkatêjê, } \\
\text { da Reserva de Mãe Maria, } \\
\text { Pará. }\end{array}$} \\
$\begin{array}{l}\text { Tavares et } \\
\text { al., 2002 }\end{array}$ & $\begin{array}{l}\text { Estudo } \\
\text { Transversal }\end{array}$ &
\end{tabular}

Indígenas moradores das três aldeias Guaraní-Mbyá, Rio de Janeiro.

Cardoso et
al., 2001 $\quad \begin{gathered}\text { Estudo } \\ \text { Transversal }\end{gathered}$

Ao longo de uma década, a população apresentou elevação dos valores basais de glicose, triglicerídeos, colesterol total, pressão arterial e índice d emassa corpórea.

Mais de $60 \%$ dos indivíduos apresentava sobrepeso, obesidade abdominal e hipertensão arterial estavam presentes em $40 \%$ da população, Dislipidemia estava presente em mais de $90 \%$ dos homens. Não foram detectados indivíduos com diabetes, porém mais de $5 \%$ já possuíam glicemia de jejum alterada.

As médias da população para Índice de Castelli I, índice de massa corpórea e glicemia estavam acima dos valores recomendados, lipoproteína de alta densidade-colesterol estava reduzida. A prevalência desses distúrbios se elevava com a idade, porém adultos com menos de 40 anos já possuíam altas prevalências e risco para doenças cardiovasculares.

Mais de $50 \%$ da população possui sobrepeso ou obesidade, $80 \%$ possui hipertensão arterial sistêmica, mais de $50 \%$ apresenta dislipidemia e glicemia de jejum alterada.

Mais de $10 \%$ da população possui glicemia de jejum alterada e quase $5 \%$ possui diabetes. Hipertensão e tabagismo foram fatores presentes na maioria dos indivíduos com alteração da glicemia ou da tolerância à insulina.

A população apresentava hipertrigliceridemia,

dislipidemia e tolerância à insulina, bem como fatores para risco cardiovascular estavam associados à altos níveis de homocisteína.

A população apresentou índice de massa corpórea de sobrepeso e valores de lipoproteína de alta densidade-colesterol

reduzidos. Sobrepeso e obesidade estavam presentes também em indivíduos com menos de 30 anos. cardiovasculares; sendo, estas, um reflexo da urbanização da população desta aldeia.

Apesar da maioria das variáveis não estarem fora dos valores de referência, é notável o aumento das variáveis após o processo de urbanização. Espera-se que, em mais uma década, esses valores estejam acima dos preconizados e a população possua mais distúrbios, além do sobrepeso já encontrado.

Sedentarismo e mudança nos hábitos alimentares levaram esta população a um grave perfil de alterações antropométricas e metabólicas. Apesar de diabetes não ter sido reportada, síndrome metabólica estava presente em mais de $30 \%$ da população, indicando efeitos negativos da urbanização e alerta para eventos cardiovasculares a curto e longo prazo.

Esta população se encontra em grande risco para eventos cardiovasculares e necessita de medidas intervencionistas. Etilismo e tabagismo não foram avaliados, mas podem estar associados a outros fatores advindos da urbanização nas principais causas dos distúrbios encontrados.

O tabagismo, bem como a alimentação pobre em frutas e vegetais foram os fatores mais associados com a presenta de aumento no peso corporal e aparecimento de comorbidades causadoras de eventos cardiovasculares.

Hábitos alimentares e tabagismo foram os principais fatores associados às alterações da glicemia encontradas. Há a necessidade de medidas preventivas, pois o risco de diabetes nesta população está mais relacionado com fatores ambientais.

A homocisteína é mais um componente bioquímico que se apresenta como fator para desenvolvimento de doenças cardiovasculares.

Esta população encontra-se em transição de perfil não-urbanizado para urbanizado e a mudança socioeconômica, comportamental e alimentar está levando a aumento do peso corporal e acarretando em aparecimento de doenças cardiometabólicas. 


\begin{tabular}{|c|c|c|}
\hline $\begin{array}{l}\text { Santos et } \\
\text { al., } 2012\end{array}$ & $\begin{array}{c}\text { Estudo } \\
\text { Transversal }\end{array}$ & $\begin{array}{l}\text { População de etnia Khisêdjê } \\
\text { do Médio Xingu, Mato } \\
\text { Grosso. }\end{array}$ \\
\hline $\begin{array}{l}\text { Bresan et } \\
\text { al., } 2015\end{array}$ & $\begin{array}{c}\text { Estudo } \\
\text { Transversal }\end{array}$ & $\begin{array}{l}\text { Moradores da Aldeia } \\
\text { Pinhalzinho, Terra Indígena } \\
\text { Xapecó, Santa Catarina. }\end{array}$ \\
\hline
\end{tabular}

\begin{tabular}{|c|c|c|}
\hline $\begin{array}{l}\text { Mancilha- } \\
\text { Carvalho et } \\
\text { al., } 1991\end{array}$ & $\begin{array}{c}\text { Estudo } \\
\text { Transversal }\end{array}$ & $\begin{array}{l}\text { Indígenas de etnia } \\
\text { Yanomami, de três aldeias na } \\
\text { serra de Surucucu e de três } \\
\text { aldeias na região dos rios } \\
\text { Catrimani e Ajarani, Roraima. }\end{array}$ \\
\hline $\begin{array}{l}\text { Mancilha- } \\
\text { Carvalho e } \\
\text { Silva, } 2003\end{array}$ & $\begin{array}{c}\text { Estudo } \\
\text { Transversal }\end{array}$ & $\begin{array}{l}\text { Indígenas de etnia } \\
\text { Yanomami, de três aldeias na } \\
\text { serra de Surucucu e de três } \\
\text { aldeias na região dos rios } \\
\text { Catrimani e Ajarani, Roraima } \\
\text { - comparados com grupos } \\
\text { étnicos de um estudo } \\
\text { multinacional com } \\
\text { localizações. }\end{array}$ \\
\hline
\end{tabular}

Mais de $25 \%$ da população é pouco ativa ou sedentária, Hipertensão arterial e diabetes foram pouco prevalentes, mas obesidade e dislipidemia foram encontradas em mais de $30 \%$ da população estudada. Hipertensão arterial e obesidade estavam altamente relacionados com fatores de urbanização e renda per capita elevada.

A população estudada apresentou valores de peso, pressão arterial e concentrações séricas dos íons sódio, potássio, cálcio e magnésio dentro dos valores de referência.

A população Yanomami manteve valores dentro da normalidade e um alto perfil protetor para doenças como hipertensão arterial sistêmica e obesidade, quando comparados com a população do Quênia, Nova Guiné e dos demais 48 centros avaliados.
Os Khisêdjê, antigos Suyá, mostram mudanças comportamentais com o processo de urbanização que acarretaram no aumento de pessoas com peso corporal elevado e comorbidades metabólicas.

O aumento de recursos e tecnologia, além da aquisição de alimentos industrializados geram piora nutricional e aumento do risco para doenças crônicas não transmissíveis.

O estado de saúde encontrado nesta população se deu pela baixa ingesta de sódio e gorduras saturadas e alto consumo de fibras e potássio. Assim, apresentando um perfil cardioprotetor, mesmo nos indivíduos mais velhos.

A não urbanização e isolamento deste grupo étnico manteve forte os costumes praticados nas atividades diárias e alimentares. Assim, refletindo em um perfil saudável de variáveis antropométricas e metabólicas.

Fonte: Autores.

\section{Características dos riscos cardiovasculares}

Dentre as DCNTs, as DCVs são as principais causas de morbimortalidade no Brasil e no mundo. Um terço de todos os óbitos está relacionado a esta enfermidade, que também resulta em baixa qualidade de vida e redução da expectativa de vida. DCVs têm grande impacto nos gastos em saúde, pois levam ao internamento prolongado (de um a três meses) ou, ainda, a consecutivos internamentos de curto prazo. Enumerando os principais fatores de risco que predispõem e potencializam o surgimento das DCVs, podemos citar os valores de lipídicos plasmáticos fora dos limites de referência para sexo e idade, sedentarismo e excesso de peso. Estes fatores apresentaram-se bastante elevados nas populações indígenas do Brasil e do Mundo (Brasil, 2011; Grundy et al., 1998; Simão et al., 2013). A Organização Pan-americana de Saúde (OPAS, 2016) e a Organização Mundial de Saúde (OMS, 2017) afirmam que embora fatores genéticos tenham o seu papel no desenvolvimento das DCVs, o estilo de vida do indivíduo, bem como hábitos alimentares inadequados, são determinantes para o desenvolvimento e progressão destas enfermidades, motivo pelo qual tem se observado elevada prevalência desta morbidade em indígenas brasileiros.

\section{Urbanização}

No fenômeno de urbanização das terras indígenas, tem sido encontrado um aumento do surgimento de fatores de riscos cardiovasculares e, consequentemente, do número de óbitos por DCVs (OMS, 2017). Este aumento se dá pelo maior acesso da população, até então isolada, a costumes e atividades comuns em cidades maiores, que já passaram pelo processo de urbanização e hoje vivem a globalização. Apesar de benefícios advindos da comunicação e interação intercultural, o contato com o meio externo vem trazendo malefícios aos hábitos alimentares e ao condicionamento físico (Armstrong et al., 2018), como mostra a Figura 1.

A estratificação de tribos de um estudo transversal em: grupos indígenas mais urbanizados - G2 (Tuxá, Truká e Tumbalalá) e menos urbanizados - G1 (Funi-ô, Pankararu, Kiriri e Pankararé) possibilitou a avaliação do efeito de aspectos da 
urbanização sobre a saúde das tribos. Assim, o grau de urbanização refletiu-se em maiores taxas de mortalidade cardiovasculares nas populações estudadas, cerca de $15 \%$ a mais nos grupos mais urbanizados do que nos menos urbanizados. A urbanização impacta na exposição a novos hábitos alimentares e de vida, divergentes de hábitos ancestrais, que estavam voltados à alimentação rica em frutas, verduras e consumo de peixes. Além disso, há menor necessidade de trabalho braçal por atividades de caça, pesca e agricultura de subsistência, que são extinguidos, passando a aumentar a utilização de tecnologias para otimizar trabalhos sem a utilização de força física (Favaro et al., 2007). O baixo consumo de calorias diárias pode levar ao sobrepeso e obesidade, com suas consequências metabólicas.

Figura 1. Causas de doenças cardiovasculares na população indígena brasileira de acordo com a literatura atual.

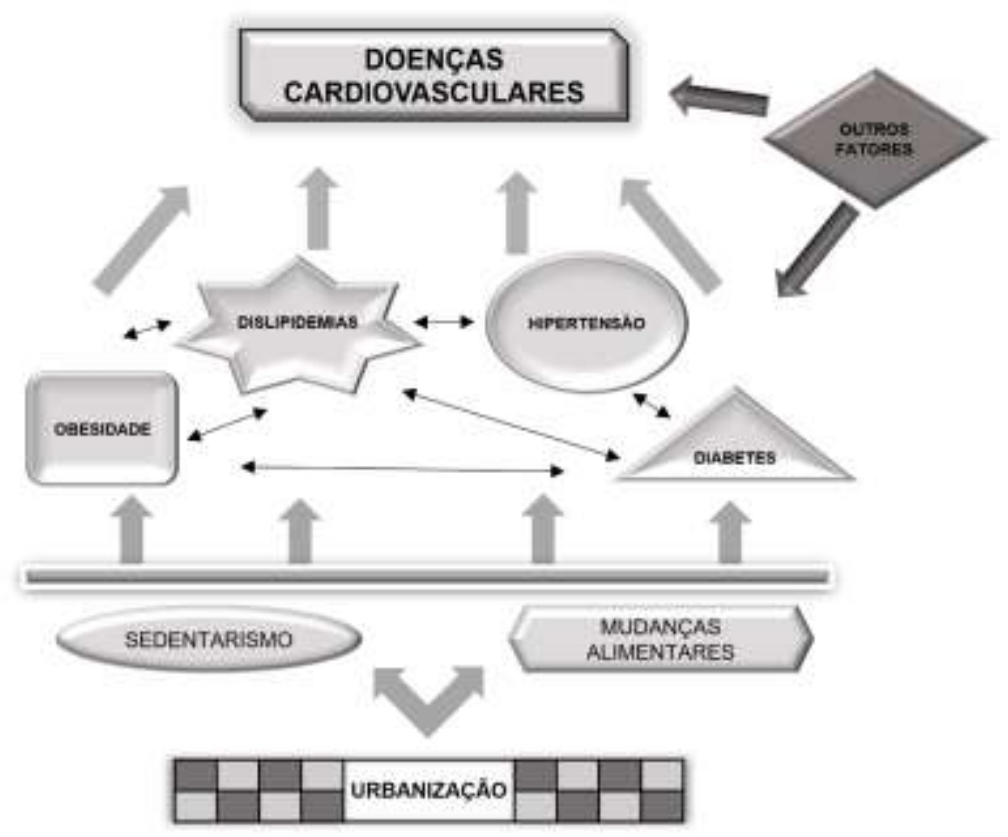

Fonte: Autores.

Vale salientar que a ocidentalização das terras indígenas - outra forma de nomear o fenômeno de urbanização - ocorre desde o período de colonização e este fato não ocorre apenas no Brasil, estudos com populações internacionais também confirmam a forte relação entre alterações do estilo de vida tradicional com o aumento da prevalência de sobrepeso, pressão arterial sistêmica e diabetes, como pode ser verificada entre os índios Pima que vivem no Estado do Arizona, Estados Unidos (Story et al., 2003). Não apenas restrito ao Arizona, a literatura também aponta o aumento de risco para eventos cardiovasculares em grupos étnicos da Austrália, Nova Zelândia e Canadá. Apesar de maior atenção das políticas públicas destes países para grupos indígenas encontrados em suas populações (Stoner et al., 2012; Calábria et al., 2018).

\section{Sedentarismo}

Devido à redução na quantidade de terras e, consequentemente, da agricultura de subsistência, passou-se a existir uma redução das atividades rotineiras dentro dos aldeamentos, como a caça, a pesca e a agricultura, forçando-os a introduzirem a economia monetária advinda do trabalho assalariado. Assim, trazendo menores esforços físicos e favorecimento à entrada de alimentos industrializados às mesas. Todas essas alterações no modo de vida desses povos trouxeram repercussões à saúde e, gradativamente, à qualidade de vida, com o surgimento e aumento crescente das DCNTs (Wirsing, 1985). Isto é visto em um estudo transversal realizado com a população indígena Khisêdjê do Parque Indígena do Xingu, no qual a prevalência de síndrome metabólica foi evidenciada nos indivíduos que, apesar de possuírem desempenho a testes de esforço satisfatórios, 
possuíam a quantidade de passos caminhados por dia menor do que os indivíduos sem síndrome metabólica (Simão et al., 2013).

Como resultado das alterações na relação dos indígenas com a terra, o modo de trabalho e a crescente adição de tecnologias reduziu a necessidade de trabalhos que utilizam de esforço físico. A introdução de tecnologia na aldeia explica, em parte, a dislipidemia e sobrepeso nessas populações, pois o uso de barco a motor na pescaria e no translado, e de trator e serra elétrica no cultivo reduz a atividade física habitual, com menor gasto de energia e deposição na forma de tecido adiposo (Salvo, et al., 2009). Deste modo, os termos obesidade, hipertensão, diabetes mellitus e dislipidemias, bem como a síndrome metabólica tornaram-se mais comuns dentro de áreas indígenas. Esses fatores são potencializados pelo consumo de alimentos industrializados ricos em carboidratos, com baixo valor proteico e de ingesta de vitaminas e sais minerais reduzida (Wirsing, 1985; Schmidt et al., 2011).

\section{Hábitos Alimentares}

Pouco se sabe sobre os hábitos alimentares das comunidades indígenas que sofrem urbanização, como levanta Coimbra Jr et desde a década de 90 (Coimbra Jr, Santos, 1991). Apesar disto, a literatura já mostra uma realidade de dificuldade da população indígena de se manter com alimentação com diversidade de fontes de nutrientes. No estudo de Coimbra Jr et al, com o grupo de 400 indígenas Suruí do estado de Rondônia, foi observado um desenvolvimento de altura e peso de crianças por volta do $3^{\circ}$ percentil, indicando déficits no desenvolvimento da altura e peso esperados para a idade. Estes dados foram relacionados com as infecções que acometem a população infantil indígena devido ao precário saneamento e pode refletir negativamente em alterações neuropsicomotoras e metabólicas futuras(Coimbra Jr, Santos, 1991).

Achados similares foram encontrados em um estudo transversal realizado 10 anos após o do grupo Suruí, com a etnia Terena localizada na Aldeia Córrego do Meio, no estado do Mato Grosso do Sul. Cerca de 8\% das crianças apresentaram déficit de peso para a idade, $16 \%$ apresentaram na estatura para a idade e $1 \%$ apresentou déficit no peso para estatura. Indicando, assim, uma alta prevalência de desnutrição crônica, o que causa maiores danos ao desenvolvimento das crianças desta população (Ribas et al., 2001). Mais recentemente, um estudo transversal com famílias indígenas Teréna, da Área Indígena Buriti do Mato Grosso apresentou apenas 24,5\% das 49 famílias entrevistadas que se declararam com segurança alimentar. As demais famílias demonstraram preocupação com a quantidade e a qualidade dos alimentos levados à mesa. Muito da preocupação encontrada nestas famílias se dá pela renda per capita de $\mathrm{R} \$ 66,44$, que apesar de haver famílias com o dobro desta renda, ainda se encontram com valores baixos para aquisição de insumos e isto leva a uma má alimentação, momentos de jejum intermitente e aquisição de produtos hipercalóricos, mas pobres em nutrientes (Favaro et al., 2007).

\section{Obesidade}

As alterações nas formas de subsistências das populações indígenas têm tido grande repercussão na saúde desses povos de forma que tem se refletido diretamente no aumento das prevalências das doenças crônicas não transmissíveis (DCNTs). Este fato tem provocado o interesse de pesquisadores em investigar as causas dessas enfermidades, com uma busca constante pelo perfil nutricional das etnias, principalmente nas populações brasileiras. O que pode ser evidenciado no trabalho de Capelli e Koifman (Capelli, Koifman, 2001), no qual se encontrou em indígenas Parkatêjê uma prevalência de sobrepeso de $23,7 \%$ em homens e $50,0 \%$ em mulheres adultas.

Gugelmin et al (2006) reportaram grande concentração da população da aldeia São José no Mato Grosso (80\%) classificada com sobrepeso ou obesa. Foi sugerido que talvez o ponto de corte do IMC não fosse o mais adequado, pois os moradores possuem estatura um pouco menor que os brasileiros que moram em terras não indígenas; no entanto, há a hipótese 
de que realmente há um processo de transição nos dados antropométricos destes indivíduos devido a mudanças alimentares e isto gera preocupação no controle dos alimentos disponíveis.

Isto também foi visto numa coorte realizada em 1999-2000 e em 2009-2010; houve um aumento dos IMCs de indígenas Khisêdjê, que já se encontravam em valores com estado de sobrepeso em 1999-2000, por volta de 23,1\% das mulheres e $25,8 \%$ dos homens. Este progresso para obesidade foi relacionado com o bom convívio da população Khisêdjê, moradora do Parque Indígena do Xingu, com os municípios vizinhos e a intensa adição de suas culturas e hábitos à sua cultura ancestral (Mazzuchetti et al., 2014). Outro estudo, desta vez transversal, com 170 indivíduos da mesma população, evidenciou obesidade central em 43\% das mulheres com mais de 20 anos e em 18\% nos homens. As mulheres tinham maior prevalência devido à paridade, porém as variáveis que indicaram síndrome metabólica nesta população estavam relacionadas principalmente com o nível de sedentarismo, independente do sexo (Simão et al., 2013). Ainda nesta população, 63,9\% apresentaram dislipidemia. Do mesmo modo, Gimeno e colaboradores (2007) observaram que 77,1\% dos índios Aruak tinham dislipidemia, indicando mais um grupo étnico indígena com altas prevalências de obesidade associada ao processo de urbanização de aldeias indígenas.

Ademais, um estudo transversal com indivíduos Suyá do Parque Indígena do Xingu (Salvo et al., 2009), verificou que dos 86 participantes da entrevista, todos com idade acima de 20 anos, 46,5\% possuíam sobrepeso (maioria de homens) e 38,4\% tinham obesidade central (maioria em mulheres). $\mathrm{O}$ autor afirma que a obesidade central maior nas mulheres Suyá se explica pelo número de gestações que é elevado entre elas, porém o sobrepeso encontrado na população geral, corroborando com os demais estudos, se dava por mudança nos hábitos de vida, em especial, na má alimentação e na falta de atividade física.

A literatura aponta para um aumento de sobrepeso em outro grupo indígena, os Xavantes da Aldeia São José, e que esta elevação se correlaciona com o tempo e contato com não índios, pois, quando se compara dados antropométricos coletados nesses povos na década de 1960 com outros dados da mesma população coletado na década de 1990, verifica-se que os valores médios de peso são superiores em 5,7 - 8,3kg em homens e 13,7 - 17,6kg em mulheres. Tal fato pode ser comprovado por meio da investigação de outra comunidade indígena de mesmo grupo étnico, como a comunidade moradora de Pimentel Barbosa, cujas formas de subsistência não sofreram alterações para o estilo de vida como na comunidade de São José. Enquanto a prevalência de obesidade em São José era bastante elevada, 24,6\% em homens e 41,3\% em mulheres, os achados em Pimentel Barbosa apontam prevalências de 2,5\% em homens e 4,8\% em mulheres (Gugelmin, Santos, 2006).

Um dos fatores limitantes no processamento e comparação dos resultados reside no fato de que são utilizadas metodologias diferentes nesses estudos. É importante ressaltar que os povos indígenas ainda não foram incluídos em importantes instrumentos de aferição e investigação do estado nutricional atual de populações feitos por meio de grandes inquéritos nacionais como a Pesquisa Nacional sobre Saúde e Nutrição (PNSN) de 1989, a Pesquisa Nacional sobre Demografia e Saúde (PNDS) de 1996 e a Pesquisa sobre Padrões de Vida (PPV) de 1997, os quais podem fornecer importantes dados epidemiológicos relacionados ao perfil nutricional desses nativos brasileiros para que se compreenda melhor as mudanças na aquisição e distribuição de massa e as enfermidades decorrentes de alterações antropométricas e metabólicas.

\section{Diabetes}

Assim como a obesidade, estudos mostram a presença de diabetes em populações indígenas do Brasil, porém a grande parte de sua prevalência está associada a outros distúrbios, como dislipidemias. A hiperglicemia foi encontrada em 70,2\% das mulheres e 46,8\% dos homens da população Xavante, valores maiores do que na população brasileira, indicando risco três vezes maior de vulnerabilidade desta população a eventos cardiovasculares (Soares et al., 2018). Apesar disto, a maioria dos estudos mostra menor prevalencia de hiperglicemia do que dislipidemia e outros distúrbios metabólicos, como na população Khisêdjê do Parque Indígena do Xingu, que não apresentou valores fora dos níveis de referência para glicemia na coorte 
realizada em 1999-2000 e 2009-2010. Embora tenha sido notada elevação da glicemia basal, que pode vir a aumentar cada vez mais, a ponto de evoluir à diabetes, caso não haja mudanças no estilo de vida (Mazzuchetti et al., 2014).

Além disso, um estudo transversal com 150 indivíduos das etnias Kaigang e Guarani moradores de Nanoai e Porto Alegre evidenciou uma prevalencia de 65,3\% dos indivíduos com síndrome metabólica e, destes, 86\% apresentavam hiperglicemia, indo de encontro com os $14 \%$ que apresentavam hiperglicemia e não possuíam síndrome metabólica (Rocha, 2018). Isto indica uma predisposição maior a alterações lipídicas e antropométricas previamente a distúrbios no metabolismo da insulina e glicose. Em outros dois estudos, com a etnia Aruák do Alto Xingu (Gimeno et al., 2007) e com a população da Aldeia Jaguaripu no estado do Mato Grosso do Sul (Oliveira et al., 2011), a glicemia de jejum se apresentou alterada em menos de $10 \%$ da população, e, diabetes mellitus estava diagnosticada em menor porcentagem. Dos habitantes com diabetes mellitus, mais de $90 \%$ possuíam hipertensão ou aumento da circunferência abdominal. Pouco se é discutido sobre a baixa prevalência de diabetes em pacientes que não possuam outros distúrbios metabólicos, porém, acredita-se que, para estas populações, a alteração na glicemia seja um evento secundário à obesidade, tabagismo e dislipidemia.

\section{Dislipidemia}

As dislipidemias são reportadas em grande prevalência nas comunidades indígenas brasileiras estudadas. Muitas vezes, os indivíduos já se encontram em estado de síndrome metabólica, o que dificulta o delineamento do processo que causou tal síndrome, porém a alimentação hipercalórica e a redução de atividade física são os fatores mais relacionados. Dentre os maiores achados, encontram-se hipercolesterolemia, redução nos níveis de colesterol da lipoproteína de alta densidade (HDL-c) e aumento nos níveis de colesterol da lipoproteína de baixa densidade (LDL-c), além de hipertrigliceridemia (Gimeno et al., 2007). Em uma coorte com os indígenas Khisêdjê do Parque Indígena do Xingú, a população já se encontrava com esses valores de lipídeos alterados, devido ao contato com municípios vizinhos ao parque e, após 10 anos, houve aumento dessas taxas, indicando uma piora do estado lipídico da população.

Em outra etnia encontrada no Parque Indígena do Xingu, a Suyá, um estudo transversal reportou que a dislipidemia estava presente em $45,8 \%$ das mulheres abaixo dos 40 anos e em $72 \%$ dos homens na mesma faixa etária. Estes valores mais evidentes na população masculina foram relacionados ao aumento de tecnologias, que resulta no sedentarismo. As mulheres sofreram com mudanças alimentares, no entanto os autores sugerem que o fato de permanecerem em atividades do lar as mantiveram ativas o suficiente para não elevar de forma tão expressiva quanto na população masculina, apesar de também terem distúrbios metabólicos associados à multiparidade (Salvo et al., 2009). Outrossim, a prevalência de dislipidemia foi vista em 44,4\% dos indivíduos da etnia Parkatêjê no sudeste do estado do Pará, não havendo discrepâncias entre os valores para homens e para mulheres. Neste caso, esta comunidade não possuía grande contato com o ambiente externo e não havia grandes fatores de urbanização na aldeia, o que reduz a possibilidade de fatores causadores de dislipidemias, como os já vistos em outros estudos, como tecnologias para o trabalho e produtos industrializados (Tavares et al., 2002).

Por outro lado, Cardoso e colaboradores (Cardoso, Mattos, Koifman, 2001), em um estudo transversal com 80 homens e 71 mulheres membros da população indígena Guaraní-Mbyá, encontrou resultados diferentes das demais nos valores dos lipídios. A média dos valores de colesterol total, LDL-c e triglicerídios estavam inferiores aos valores de referência, enquanto as médias de HDL-c, para os dois sexos, se encontravam dentro dos valores de referência. Pouco foi discutido, mas se acredita que os resultados foram reflexo de uma dieta hipocalórica e níveis regulares de atividade física semanal.

\section{Hipertensão}

Em relação aos níveis de pressão arterial, os indígenas Khisêdjê apresentaram valores de pressão arterial sistólica (PAS) e diastólica (PAD) dentro da faixa de normalidade. No entanto, assim como a glicemia, houve um aumento significativo 
da PAS em 15mmHg nas mulheres e $10 \mathrm{mmHg}$ nos homens no segundo momento de avaliação da coorte, porém não ultrapassaram os valores de referência, mostrando um aumento na tendência desta população a possuírem fatores para eventos cardiovasculares com o passar dos anos e interação com comunidades não indígenas (Mazzuchetti et al., 2014). Em nenhum dos demais estudos com comunidades indígenas foi encontrado situação semelhante, pois, na maioria dos trabalhos, existe sempre uma forte relação das alterações da PAS com outros fatores de risco das DCVs (Tavares et al., 2002; Cardoso, Mattos, Koifmann, 2001; Santos et al., 2012; Bresan, Bastos, Leite, 2015).

Um estudo transversal (Bresan, Bastos, Leite, 2015), que avaliou a prevalência de níveis tensionais sugestivos de hipertensão em indígenas Kaingang, encontrou uma prevalência de hipertensão em 53,2\% nos homens e 40,7\% nas mulheres de uma população de 355 indivíduos adultos. Como fatores de correlação, a circunferência da cintura e o IMC foram as variáveis que mais demonstraram ligação com a PAS elevada. Isto também foi observado em um estudo com a etnia Kaingang de Xapecó ${ }^{32}$. Mais de $70 \%$ dos pacientes reportados como obesos apresentavam hipertensão arterial. Estudos afirmam que indígenas isolados no Brasil apresentavam pressão arterial baixa, antes do contato com a frente de expansão do desenvolvimento nacional, o que contribui para a compreensão da relação entre o modo de vida tradicional indígena e os fatores de risco cardiovasculares baixos ou ausentes (Mancilha-Carvalho et al., 1991; Mancilha-Carvalho, Silva, 2003).

\section{Outros Fatores}

Ademais às comorbidades com risco às DCVs, um estudo transversal com indivíduos da etnia Parkatêjê no sudeste do estado do Pará evidenciou hiper-homocisteinemia em aproximadamente $40 \%$ da população. Apesar deste resultado estar relacionado à resistência à insulina, para esta população, não foram encontradas correlações com a glicemia, muito menos com o teste de tolerância à insulina. No entanto, dislipidemia e hiperuricemia foram altamente correlacionadas e a hiperhomocisteinemia estava intimamente associada com comprometimento renal, que, por sua vez, foi relacionado à estado crônico de alteração metabólica (Tavares et al., 2002).

Outro fator muito associado à DCVs, independente do sexo, foi o tabagismo. Tabagismo ou histórico de tabagismo em indivíduos das etnias Kaigang e Guarani moradores de Nanoai e Porto Alegre estava altamente relacionado com a presença de síndrome metabólica. O que potencializa os riscos para eventos cardiovasculares (Rocha et al., 2011).

\section{Considerações Finais}

Apesar de escassa, a literatura possui fortes evidências do aparecimento de alterações metabólicas e doenças crônicas não infecciosas que podem gerar eventos cardiovasculares na população indígena do Brasil. Esta mudança no perfil de patologias destes grupos se dá pela redução de atividades agrícola e de caça, tendência da população a se tornar sedentária, alimentação com produtos industrializados e hipercalóricos, resultantes do processo de urbanização. Diabetes mellitus sem comorbidades é de prevalência pequena, porém hipertensão, obesidade e dislipidemias são bastante prevalentes e geram grande incidência de síndrome metabólica, que, por sua vez, ajuda no aumento de casos de diabetes mellitus, como demonstrados na figura 1. Assim, são necessários mais estudos para analisar outros fatores de riscos associados às DCVs nessas populações e existe necessidade de elaboração de estratégias para prevenir e controlar os fatores de risco já evidentes para as doenças cardiovasculares, haja vista, a população indígena no Brasil ser bastante carente de recursos e instrução.

\section{Agradecimentos}

Os autores agradecem às agências de fomento: Conselho Nacional de Desenvolvimento Científico e Tecnológico (CNPq, Proc. 312675/2018-6), Coordenação de Aperfeiçoamento de Pessoal de Nível Superior (CAPES - Código de Financiamento 001) e Fundação de Amparo à Ciência e Tecnologia do Estado de Pernambuco (FACEPE). 


\section{Referências}

Armstrong, A. C., et al. (2018) Urbanization is Associated with Increased Trends in Cardiovascular Mortality Among Indigenous Populations: the PAI Study. Arq Bras Cardiol. 110, 3, 240-245.

Brasil. Ministério da Saúde. Secretaria de Vigilância em Saúde. (2011) Departamento de Análise de Situação de Saúde. Plano de ações estratégicaspara o enfrentamento das doenças crônicas não transmissíveis (DCNT) no Brasil 2011-2022. Brasília.

Bresan, N. D, et al. (2015) Epidemiology of high blood pressure among the Kaingang people on the Xapecó Indigenous Land in Santa Catarina State, Brazil, 2013. Cad. Saúde Pública, 31, 2, 1-14.

Calábria, B., et al. (2018) Absolute cardiovascular disease risk and lip-lowering therapy among aboriginal and torres strait inslander australians. MJA, 209, 1, p.35-41.

Capelli, J. C. S., \& Koifman, S. (2001) Evaluation of the nutritional status of the Parkatêjê indigenous community in Bom Jesus do Tocantins, Pará, Brazil. Cad. Saúde Pública, 17, 2, 433-437.

Cardoso, A. M., et al. (2001) Prevalence of risk factors for cardiovasculardisease in the Guaraní-Mbyá population of the State of Rio de Janeiro. Cad. Saúde Pública, 17, 2, 345-354.

Coimbra Jr, C. E. A., \& Santos, R. V. (1991) Avaliação do estado nutricional num contexto de mudança sócio-econômica: o grupo indígena Suruí do estado de Rondônia, Brasil. Cadernos de Saúde Pública, 7, 4, 538-562.

Coimbra Jr, C. E. A., \& Santos, R. V. (2000) Saúde, minorias e desigualdade: algumas teias de inter-relações, com ênfase nos povos indígenas no Brasil. Ciência e Saúde Coletiva, 5, 1, 125-132.

Favaro, T, et al. (2007) Segurança alimentar em famílias indígenas Terena, Mato Grosso do Sul, Brazil. CAD Saúde Pública, $23,4,785-793$.

Gimeno, S. G. A, et al. (2007) Metabolic and anthropometric profile of Aruák Indians: Mehináku, Waurá and Yawalapití in the Upper Xingu, Central Brazil, 2000-2002. Cad. Saúde Pública, 23, 8, 1946-1954.

Grundy, S. M., et al. (1998) Primary prevention of coronary heart disease: guidance from Framingham:a statement for healthcare professionals from the AHA task force on risk reduction. Circulation, 97, 18, 1876-87.

Gugelmin, A S., \& Santos, R. V. (2006) Uso do índice de massa corporal na avaliação do estado nutricional de adultos indígenas Xavante, terra indígena Sangradouro-Volta Grande, Mato Grosso, Brasil. Cad Saúde Pública, 22, 9, 1865-1872.

Leite, M S., et al. (2007) Gugelmin SA. Alimentação e nutrição dos povos indígenas no Brasil. In: Kac G, Sichieri R, Gigante DP, organizadores. Epidemiologia nutricional. Rio de Janeiro: Editora Fiocruz/Editora Atheneu, 503-17

Mancilha-Carvalho, J. J., et al. (1991) Blood pressure in Yanomami villages. Arq Bras Cardiol, 56, 6, 477-82.

Mancilha-Carvalho, J. J., \& Silva, N. A. S. (2003) The Yanomami Indians in the INTERSALT Study. Arq Brás Cardiol, 80, 3, $289-300$.

Mazzuchetti L, et al. (2014) Incidência de síndromemetabólica e doenças associadas na população indígena Khisêdhê do Xingu, Brasil Central, noperíodo de 1999-2000 e 2010-2011. Cad Saúde Pública, 30, 11, 1-11.

Oliveira, G. F., et al. (2011) Prevalência de diabetes melito e tolerância à glicose diminuída nos indígenas da Aldeia Jaguapiru, Brasil. Ver Panam Salud Publica, 29, 5, 315-321.

Organização Panamericana de Saúde (2016) "Social determinants and risks for health, chronic noncommunicable diseases and mental health: cardiovascular diseases," OPAS, Washington, DC, USA, http://www.paho.org

Ribas, D. L. B., et al. (2001) Nutrição e saúde infantil em uma comunidade indígena Terena, Mato Grosso do Sul, Brasil. Cad Saúde Pública, 17,2 , 323-331.

Rocha AKS, et al. (2011) Prevalência da síndrome metabólica em indígenas commais de 40 anos no Rio Grande do Sul, Brasil. Ver Panam Salu Publica, 29, $1,41-45$.

Salvo, A. V. L. M., et al. (2009) Metabolicandanthropometric profile of Suyá. Xingu Indigenous Park, Central Brazil. Rev Bras Epidemiol, 12,3 , 458-68.

Santos, K. M., et al. (2012) Degree of physical activity and metabolic syndrome: a cross-sectional study among the Khisêdjê group in the Xingu Indigenous Park, Brazil. Cad. Saúde Pública, 28, 12, 2327-2338.

Santos, R. V., \& Coimbra Jr. C. E. A. (2003) Cenários e tendências da saúde e da epidemiologia dos povos indígenas do Brasil. In: Coimbra Jr. CEA, Santos $\mathrm{RV}$, Escobar AL, organizadores. Epidemiologia e saúde dos povos indígenas no Brasil. Rio de Janeiro: Editora Fiocruz/ABRASCO, $13-47$.

Sartori Jr, D., \& Leivas, G. C. (2017) O direito à saúde dos povos indígenas e o paradigma do reconhecimento. Direito e Práxis revista, 8, 1 , 86-117.

Schmidt, M. I., et al. (2011) Chronic non-communicable diseases in Brazil: burden and current challenges. Lancet, 377, $1949-61$.

Simão, A. F., et al; (2013) Sociedade Brasileira de Cardiologia. [I Brazilian Guidelines for cardiovascular prevention]. Arq Brás Cardiol, 101, 6, Suppl 2, 163.

Soares, L. , et al. (2018) Risco cardiovascular na população indígena xavante. Arq Brás Cardiol, 110, 6, 542-550.

Stoner, L., et al. (2012) Preventing a cardiovascular disease epidemic among indigenous populations through lifestyle changes. Int J Prev Med, 3, 4, 230-240. 
Research, Society and Development, v. 10, n. 9, e38610918254, 2021

(CC BY 4.0) | ISSN 2525-3409 | DOI: http://dx.doi.org/10.33448/rsd-v10i9.18254

Story, M., et al. (2003) Obesity in American-Indian children: prevalence, consequences and prevention. Prev Med, 37, 6, 3-12.

Tavares, E. F., et al. (2002) Relação da homocisteinemia com a sensibilidade à insulina e com fatores de risco cardiovascular em um grupo indígena brasileiro. Arq Brás Endocrinol Metab, 46, 3, 260-268.

Tavares, F. G., et al. (2012) Níveis tensionais de adultos indígenas Suruí, Rondônia, Brasil. Ciênc. Saúde Coletiva, 18, 1, 399-409.

Welch, J. R., et al. (2009) Nutrition transition, socioeconomic differentiation, and gender among adult Xavante Indians, Brazilian Amazon. Hum Ecol, 37, 1326.

Wirsing, R. L. (1985) The health of traditional societies and the effects of acculturation. Curr Anthropol, 26, 303-22.

World Health Organization. (2017) "Fact sheets: the top 10 causes of death," World Health Organization, Geneva, Switzerland, http://www.who.int/mediacentre/factsheets/fs310/en 